\title{
The Distinct Role of Tcfs and Lef1 in the Self-Renewal or Differentiation of Mouse Embryonic Stem Cells
}

\author{
Sewoon Kim ${ }^{1, *}$, Hanjun $\mathrm{Kim}^{2, *}$, Anderson Tan ${ }^{1, *}$, Yonghee Song ${ }^{1}$, \\ Hyeju Lee ${ }^{1}$, Qi-Long Ying ${ }^{3}$, Eek-hoon Jho ${ }^{1}$ \\ ${ }^{1}$ Department of Life Science, University of Seoul, Seoul, Korea \\ ${ }^{2}$ Asan Institute for Life Sciences, Seoul, Korea \\ ${ }^{3}$ Eli and Edythe Broad Center for Regenerative Medicine and Stem Cell Research at USC, \\ Keck School of Medicine, University of Southern California, Los Angeles, CA, USA
}

\begin{abstract}
Background and Objectives: Tcfs and Lef1 are DNA-binding transcriptional factors in the canonical Wnt signaling pathway. In the absence of $\beta$-catenin, Tcfs and Lef1 generally act as transcriptional repressors with co-repressor proteins such as Groucho, CtBP, and HIC-5. However, Tcfs and Lef1 turn into transcriptional activators during the interaction with $\beta$-catenin. Therefore, the activity of Tcfs and Lef1 is regulated by $\beta$-catenin. However, the intrinsic role of Tcfs and Lef1 has yet to be examined. The purpose of this study was to determine whether Tcfs and Lef1 play differential roles in the regulation of self-renewal and differentiation of mouse ES cells.

Methods and Results: Interestingly, the expression of Tcfs and Lef1 was dynamically altered under various differentiation conditions, such as removal of LIF, EB formation and neuronal differentiation in N2B27 media, suggesting that the function of each Tcf and Lef1 may vary in ES cells. Ectopic expression of Tcf1 or the dominant negative form of Lef1 (Lef1-DN) contributes to ES cells to self-renew in the absence of leukemia inhibitory factor (LIF), whereas ectopic expression of Tcf3, Lef1 or Tcf1-DN did not support ES cells to self-renew. Ectopic expression of either Lef1 or Lef1-DN blocked neuronal differentiation, suggesting that the transient induction of Lef1 was necessary for the initiation and progress of differentiation. ChIP analysis shows that Tcfl bound to Nanog promoter and ectopic expression of Tcfl enhanced the transcription of Nanog.

Conclusions: The overall data suggest that Tcf1 plays a critical role in the maintenance of stemness whereas Lef1 is involved in the initiation of differentiation.
\end{abstract}

Keywords: Tcf/Lefl, Wnt, ES cell, Self-renewal, Differentiation

Received: March 18, 2020, Revised: May 8, 2020,

Accepted: May 8, 2020, Published online: June 30, 2020

Correspondence to Eek-hoon Jho

Department of Life Science, University of Seoul, 163 Seoulsiripdae-ro,

Dongdaemoon-gu, Seoul 02504, Korea

Tel: +82-2-6490-2671, Fax: +82-2-6490-2664

E-mail: ej70@uos.ac.kr

*These authors contributed equally to this work

(a) This is an open-access article distributed under the terms of the Creative Commons Attribution Non-Commercial License (http://creativecommons.org/ licenses/by-nc/4.0/), which permits unrestricted non-commercial use, distribution, and reproduction in any medium, provided the original work is properly cited.

Copyright (c) 2020 by the Korean Society for Stem Cell Research

\section{Introduction}

Embryonic stem (ES) cells undergo self-renewal and can be differentiated into three germ layers: ectoderm, mesoderm, and endoderm. Thus, ES cells have been considered as useful tools for both research and regenerative medicine $(1,2)$. The regulators of ES cell self-renewal have been identified. Leukemia inhibitory factor (LIF), the factor secreted by feeder cells, is necessary for self-renewal of mouse ES cells in feeder cell-free condition (3), but not in human ES cells $(1,2,4)$. Knock-out experiments showed that Oct4 (5), Sox2 (6), and Nanog $(7,8)$ transcription fac- 
tors are required for self-renewal of ES cells. While increasing Oct4 expression induced differentiation of endoderm (9), the overexpression of Nanog was sufficient to sustain ES cell self-renewal in the absence of $\operatorname{LIF}(7,8)$.

$\mathrm{Wnt} / \beta$-catenin signaling has been shown to play an important role in various stem and progenitor cells $(10,11)$. In ES cells, the aberrant activation of Wnt $\beta$-catenin signaling maintains stemness of ES cells by blocking cell differentiation. While ectopic expression of secreted frizzled related protein 2 (sFRP2), an antagonist of Wnt signaling, induces enhanced neural differentiation, Wntl overexpression prevents neural differentiation (12). Stabilization of $\beta$-catenin by adenomatous polyposis coli (APC) mutation or ectopic expression of a dominant active form of $\beta$-catenin resulted in inhibition of differentiation (13, 14). Furthermore, the activation of Wnt signaling by a pharmacological glycogen synthase kinase-3 (GSK3)-specific inhibitor leads to maintenance of self-renewal in human and mouse ES cells $(15,16)$. However, evidence supporting Wnt signaling in ES cells is disputed. The $\beta$ catenin-mediated TOP activity in undifferentiated human ES (hES) cells is lower than in differentiated cells and treatment of Wnt3a leads to enhanced differentiation of hES cells in the absence of an anti-differentiation factor (17). In addition, canonical Wnt signaling regulates differentiation into mesodermal lineages or neural precursors (18-22). This contradictory response of ES cells upon Wnt signaling suggests the need for further studies to understand the regulation of ES cells by Wnt signaling.

Stemness and differentiation of ES cells are regulated by a number of transcription factors. Because (T-cell factors) Tcfs and (lymphoid enhancer factor 1) Lefl are transcription factors transmitting Wnt signal, it is possible that Tcfs and Lef1 differentially regulate self-renewal or differentiation of ES cells upon Wnt signaling. The Tcfs/ Lef1 family is composed of four genes in vertebrates (Tcfl, Lef1, Tcf3, and Tcf4) (23). Tcfs/Lef1 consists of four domains in common: a $\beta$-catenin binding domain, a central domain, a HMG DNA-binding domain, and a C-terminal tail. A variety of Tcfs/Leflisoforms are generated by alternative splicing and promoter usage $(24,25)$. At low levels of stabilized $\beta$-catenin, Tcfs/Lef1 proteins act as transcriptional repressors along with corepressor proteins, such as Groucho, C-terminal binding protein (CtBP), hypermethylated in cancer 1 (HIC1), and hypermethylated in cancer 5 (HIC5). In the presence of Wnt stimulation, however, Tcfs and Lefl act as transcriptional activators when $\beta$-catenin displaces Groucho $(24,26)$. Knockout studies of Tcfs and Lef1 genes showed that Tcfs and Lef1 exhibit redundant and non-redundant functions in embry- onic development and adult stem cell regulation (25). These diverse functions of Tcfs and Lefl suggest important roles in Wnt signaling in ES cells. Although it has been known that Tcf3 acts as a limiting factor in self-renewal of ES cells $(11,27-31)$, the role of other Tcfs and Lef1 in ES cells remains unknown.

In this study, we show that the expression of Tcfs and Lefl proteins in ES cells changes dynamically and the differential pattern under the three conditions of differentiation suggests that each Tcfs and Lef1 may act differently in ES cells. Ectopic expression of Tcfl contributes to their self-renewal and inhibits their differentiation even in the absence of LIF by sustaining the level of Nanog. In contrast, the overexpression of Lef1 does not result in self-renewal and knockdown of Lef1 inhibits differentiation. Overall, our data suggest that each Tcfs and Lef1 has a specific role in the maintenance of stemness and differentiation of ES cells.

\section{Materials and Methods}

\section{Culture and differentiation of mouse ES cells}

A6P10 mES cells (a gift from Dr. Chyuan-Sheng Lin, Columbia University, USA) and 46C mES cells (ES cell line in which EGFP was replaced into the open reading frame of Soxl gene, provided by Dr. Qilong Ying, University of Southern California, USA) were cultured in ES medium (DMEM (Gibco) with 15\% FBS, $2 \mathrm{mM}$ GlutaMAX (Gibco), MEM nonessential amino acids, $\beta$ mercaptoethanol (Gibco), tylosin, 1\% Pen/Strep (Gibco)) supplemented with LIF (Chemicon) on $0.2 \%$ gelatin-coated dishes. To induce neuronal differentiation, 46C cells were cultured in N2B27 medium (DMEM/F12 (Gibco), Neurobasal medium (Gibco), N2 supplement (Invitrogen), B27 supplement (Invitrogen), $1 \mathrm{mM}$ GlutaMAX (Gibco), $0.1 \mu \mathrm{M}$ $\beta$-mercaptoethanol (Gibco), 1\% Pen/Strep (Gibco)) on $0.2 \%$ gelatin-coated tissue culture dish (Falcon). N2B27 medium was changed every 2 days. Embryoid body (EB) formation was induced by hanging drop method. Briefly, $20 \mu 1$ drops (including 600 cells) of dissociated ES cells with ES medium plus 20\% FBS were placed on inverted lids of petri-dish (Falcon), which was filled with $3 \mathrm{ml}$ PBS. After incubation for 3 days, EB was plated on a $0.2 \%$ gelatin-coated dish in ES medium supplemented with 20\% FBS. The medium was changed every 2 days.

\section{Plasmids and transfection}

RNA obtained from a mixture of undifferentiated and differentiated mES cells was used to clone Tcf1, Lef1, Tcf3, and Tcf4. Wild-type and dominant negative forms of Tcfs/ 
Lef1 were inserted into the pCS2-HA3 vector. HA-tagged Tcfs and Lef1 were transferred into the pCAG-1 vector (modified from pPCAGIZ vector). The shRNA targeting sequences against mouse Lefl were designed using the web tool from Promega.

Sense (5'-GATCCCCGACTTAGCCGACATCAAGTTTCA AGAGAACTTGATGTCGGCTAAGTCTTTTTGGAAA-3') and antisense (5'-AGCTTTTCCAAAAAGACTTAGCCGA CATCAAGTTCTCTTGAAACTTGATGTCGGCTAAGTC GGG-3') oligonucleotides were annealed and ligated into the BglII and HindIII sites of the pSUPER.retro.puro vector (Oligoengine). HA-tagged Tcfs and Lefl plasmids were electroporated by Amaxa Nucleofector according to the manufacturer's protocol and then selected with $50 \mu \mathrm{g} / \mathrm{ml}$ Zeocin (Invitrogen). The shLef1 plasmid was electroporated by Amaxa Nucleofector technology ${ }^{\mathrm{TM}}$ and selected with $1 \mu \mathrm{g} / \mathrm{ml}$ puromycin (Sigma).

\section{Western blotting and antibodies}

ES cells were lysed in lysis buffer $(20 \mathrm{mM}$ Tris- $\mathrm{HCl}$, $\mathrm{pH}$ 7.5, $150 \mathrm{mM} \mathrm{NaCl}, 0.5 \%$ Triton X-100, $50 \mathrm{mM} \mathrm{NaF}$, $2 \mathrm{mM}$ EDTA, $100 \mu \mathrm{M}$ Na-orthovanadate, $1 \mathrm{mM}$ PMSF, $5 \mu \mathrm{g} / \mathrm{ml}$ leupeptin, and $1 \mu \mathrm{M}$ pepstatin A). The lysates were centrifuged at $13,000 \mathrm{rpm}$ for $15 \mathrm{~min}$ at $4^{\circ} \mathrm{C}$ and the supernatant was collected and used for Western blotting. Bradford (Bio-Rad) reagent was used to measure the quantity of protein. Equal amounts of protein were boiled in Laemmli sample buffer and resolved via SDS-PAGE followed by transfer to a PVDF membrane (Pall). Anti- $\beta$ actin (Sigma), anti-TCF1 (Cell Signaling), anti-LEF1 (Cell Signaling or Santa Cruz Biotechnology), anti-TCF3 (Santa Cruz Biotechnology), anti-TCF4 (Santa Cruz Biotechnology) antibodies were used as primary antibodies.

\section{Alkaline phosphatase (AP) staining}

ES cells were plated layered on a 12-well plate and cultured with or without LIF. After washing twice with PBS, cells were fixed with $4 \%$ paraformaldehyde for $10 \mathrm{~min}$ at room temperature followed by PBS washing several times. $\mathrm{AP}$ staining was performed with NBT/BCIP (4-nitro blue tetrazolium chloride, Roche; 5-Bromo4-chloro-3-indolylphosphate, Roche) staining buffer (0.1 M Tris, $\mathrm{pH}$ 9.5, 100 $\mathrm{mM} \mathrm{NaCl}, 5 \mathrm{mM} \mathrm{MgCl} 2$ ) for $15 \mathrm{~min}$ in the dark.

\section{Chromatin immunoprecipitation (ChIP) assay}

Cells were cross-linked with $1 \%$ formaldehyde (Sigma) at room temperature for $10 \mathrm{~min}$ with gentle shaking and then incubated in $0.125 \mathrm{M}$ glycine for $5 \mathrm{~min}$ with gentle shaking. Cells were washed twice with ice-cold PBS before harvest. Re-suspended cells with hypotonic buffer $(10 \mathrm{mM}$
Hepes-KOH, pH 7.8, $10 \mathrm{mM} \mathrm{KCl}, 1.5 \mathrm{mM} \mathrm{MgCl} 2$ ) were swollen on ice for $10 \mathrm{~min}$ and passed through a 26.5-gauge needle 6 times. After centrifugation at $1000 \mathrm{~g}$ for $5 \mathrm{~min}$ at $4^{\circ} \mathrm{C}$, the pellets were incubated in nuclei lysis buffer (1\% SDS, $50 \mathrm{mM}$ Tris-HCl, $\mathrm{pH}$ 8.0, $10 \mathrm{mM}$ EDTA) for $10 \mathrm{~min}$ on ice with occasional vortexing. Chromatin was sheared to an average length of $0.2 \sim 1 \mathrm{~kb}$ by sonication on ice. Cellular debris was removed by centrifugation $\left(13,000 \mathrm{rpm}, 15 \mathrm{~min}, 4^{\circ} \mathrm{C}\right)$, and the concentration of supernatant was determined with a spectrophotometer. The appropriate volume of chromatin was diluted $1 / 10$ in ChIP dilution buffer $(0.01 \%$ SDS, $20 \mathrm{mM}$ Tris-HCl, $\mathrm{pH}$ 8.0, 167 $\mathrm{mM} \mathrm{NaCl}, 1.2 \mathrm{mM}$ EDTA, 1.1\% Triton X-100) followed by preclearance at $4^{\circ} \mathrm{C}$ for $2 \mathrm{~h}$ with $10 \mu 1$ protein $\mathrm{A} / \mathrm{G}$ plus-agarose beads (Santa Cruz Biotechnology). For immunoprecipitation, goat, rabbit-IgG (Bethyl), anti-Lef1 (Santa Cruz Biotechnology or Cell Signaling), anti-Tcf3 (Santa Cruz Biotechnology), anti-Tcfl (Cell Signaling) antibodies were used at $4^{\circ} \mathrm{C}$ overnight. Immunoprecipitated chromatins were eluted, and then reverse cross-linked by the addition of $0.3 \mathrm{M} \mathrm{NaCl}$ at $65^{\circ} \mathrm{C}$ overnight. Following phenol-chloroform extraction and ethanol precipitation, the DNA was dissolved in $50 \mu 1$ TE buffer $(10 \mathrm{mM}$ Tris$\mathrm{HCl}, \mathrm{pH}$ 8.0, $1 \mathrm{mM}$ EDTA). For PCR, $2 \mu 1 \mathrm{DNA}$ was used. A Nanog primer (forward: 5'-TCTGCTTATACACA GAAGCC-3' and reverse: 5'-AAGTGCCTCAGCCGTCTA AG-3') was used for PCR.

\section{Reverse transcriptase-PCR (RT-PCR)}

Total mRNA from ES cells were isolated by TRIzol (Invitrogen) according to manufacturer protocol. cDNA was made from total RNA using reverse transcriptase (Promega) with random primers. For PCR, taq polymerase (Cosmo genetech) was used and visualized by $1 \%$ of agarose gel. GAPDH or 18s rRNA were used for normalization of mRNA expressions. Primers used for RT-PCR can be found in Supplementary Table S1.

\section{Results}

\section{Differential expression of Tcfs and Lefl}

Wnt signal stimulates the self-renewal and differentiation of ES cells into various lineages. We investigated the varying response of ES cells in the presence of Wnt signal. As Tcfs and Lef1 act as the downstream effectors in Wnt signal transmission, we speculated that the differential response may result from differential function of each Tcfs and Lef1.

Prior to investigation of the function of Tcfs and Lefl, the change in Tcfs and Lef1 mRNA levels during differ- 
(A)

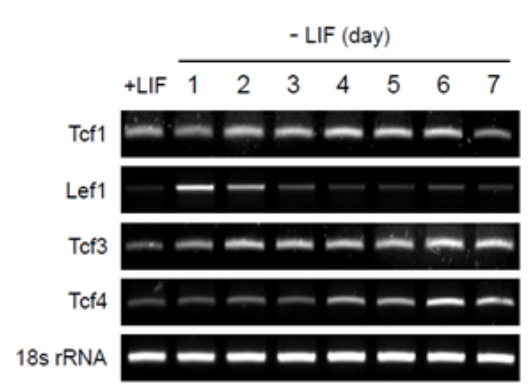

(B)

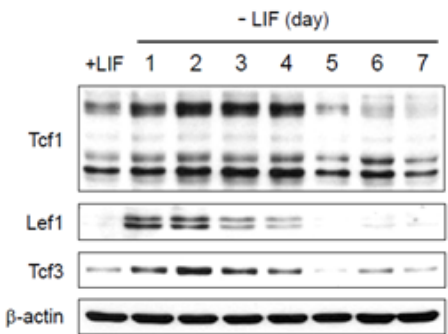

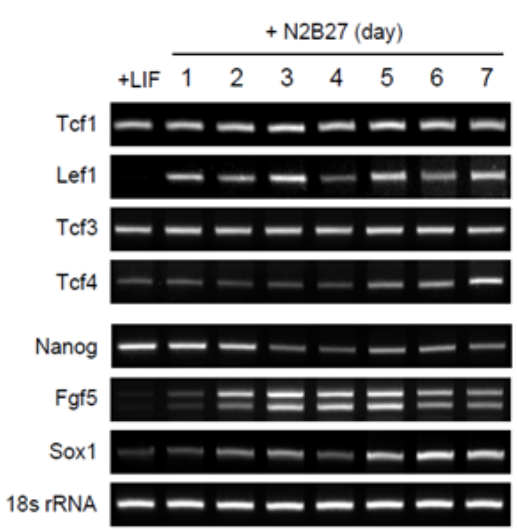
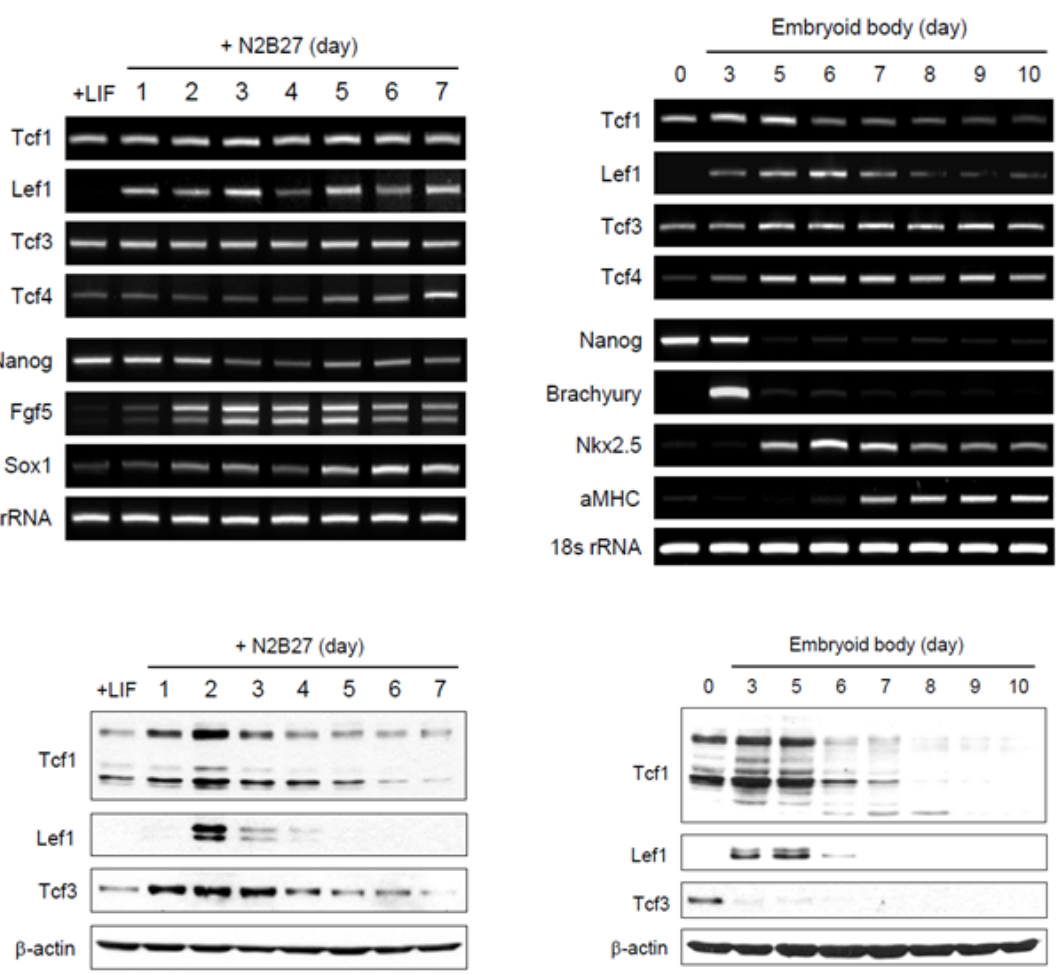

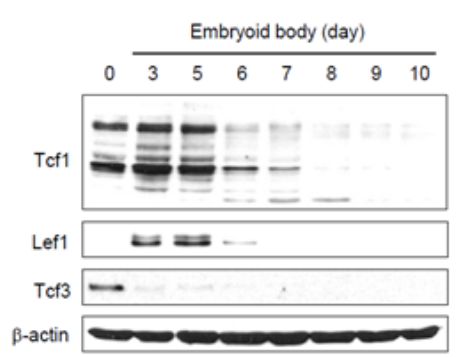

Fig. 1. The pattern of Tcfs and Lef1 expression in ES cells. (A) A6P10 ES cells were differentiated in the absence of LIF (left), in N2B27 medium without LIF (middle), or via EB formation without LIF (right). The differentiated ES cells were harvested on the days indicated. The amount of Tcfs and Lef1 transcripts was measured by RT-PCR analysis. The bands for Tcfs and Lef1 represent wild-type transcripts including $\beta$-catenin-binding domain. Self-renewal marker (Nanog), neural precursor markers (Sox1 and Fgf5) for N2B27 differentiation and mesodermal markers (Brachyury, Nkx2.5, $\alpha \mathrm{MHC}$ ) for EB differentiation were used to confirm proper differentiation; 18s rRNA was used as a loading control. (B) The endogenous protein levels of Tcfs/Lef1 were detected by western blotting. $\beta$-actin was used as a loading control.

entiation was detected by RT PCR (Fig. 1A). Under all conditions of differentiation, the Tcf3 transcript was found to be increased during the early days of differentiation. This data is consistent with previous reports that TCF3 is involved in the early stage of differentiation by suppressing pluripotent genes (32). In addition, Tcf4 transcript was also gradually increased during differentiation. Interestingly, Lefl transcript was transiently induced after the removal of LIF although this induction was maintained in N2B27 media. There was no significant change in Tcf1 expression following LIF depletion and N2B27. However, Tcf1 transcript decreased from day 6 post-EB formation. These results suggest that Lefl might play a role in the initial stages of differentiation and Tcf4 might be associated with late differentiation.

It has been known that the function of Tcfs/Lef1 is affected by post-translational modifications such as phosphorylation and sumoylation (26). Thus, we performed western blotting analysis to investigate the protein levels of Tcfs and Lef1 (Fig. 1B). The protein levels of Tcfs and
Lef1 were dynamically changed compared to the changes in Tcfs and Lefl transcripts. Similar to RT PCR data, Lef1 protein was expressed transiently at an early stage of differentiation. In contrast, the expression of Tcf1 and Tcf3 proteins revealed different patterns of mRNA levels. Under the conditions of LIF depletion or N2B27 medium, the levels of Tcf1 and Tcf3 protein were elevated until the day Lef1 protein was expressed followed by a sharp decline during subsequent differentiation. The expression pattern of Tcfl protein during EB formation was similar to that under other conditions, whereas the pattern of Tcf3 expression differed completely. After EB formation for 3 days, the loss of Tcf3 protein was inconsistent with RT PCR data, suggesting that the Tcf3 protein might be degraded by unknown mechanisms during EB formation. These results suggest that measurement of protein levels might be more appropriate for Tcfs/Lefl studies in ES cells. The variable expression of Tcfs and Lef1 proteins under differentiated and undifferentiated conditions supports our hypothesis that the role of each Tcfs and Lef1 
(A)

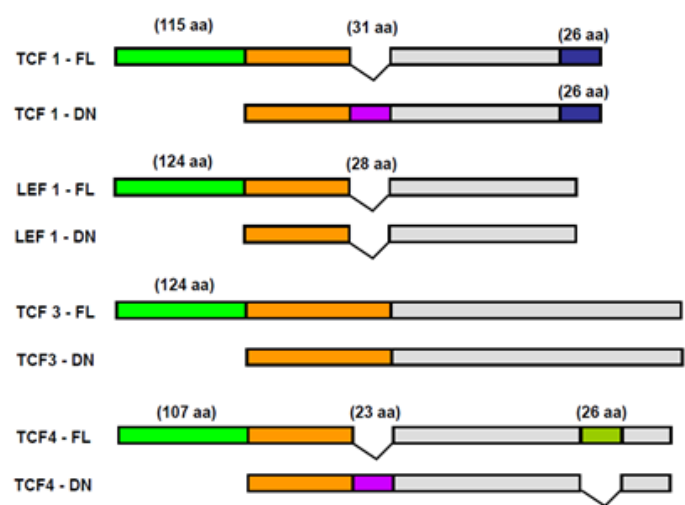

(B)

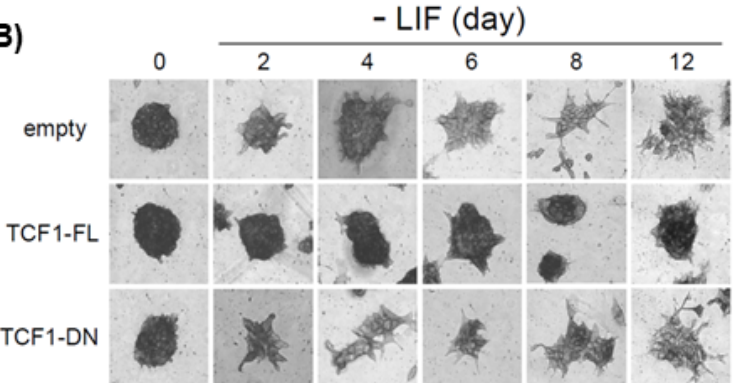

(C)

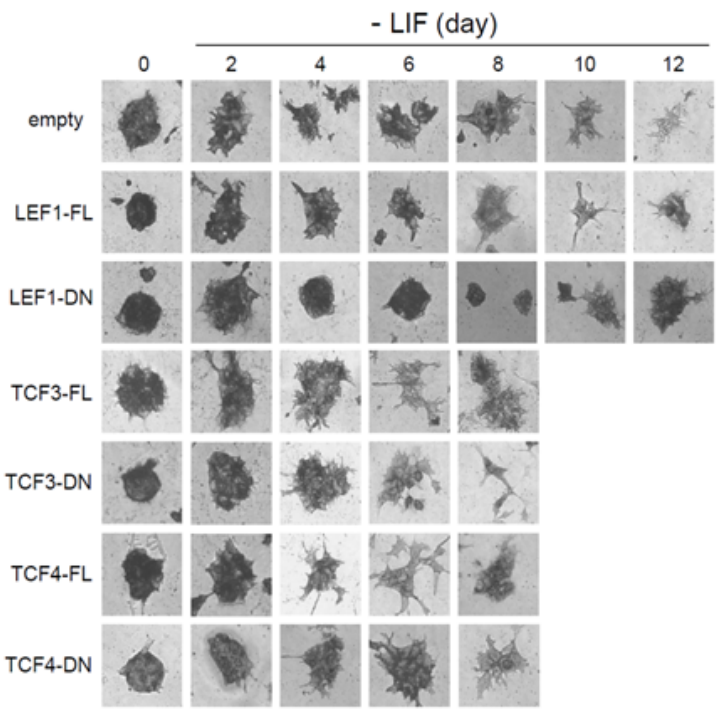

Fig. 2. The effect of ectopic expression of Tcfs and Lef1 after LIF depletion. (A) A schematic diagram of Tcfs and Lef1 constructs (FL, full length; and DN, dominant negative). (B, C) A6P10 ES cells were transfected with control vector, Tcf1 (FL or DN), Lef1 (FL or DN), Tcf3 (FL or DN), or Tcf4 (FL or DN) and then selected using Zeocin. Selected ES cells were stained with alkaline phosphatase (AP), a marker of self-renewal, on the days indicated after LIF removal.

may differ in self-renewal and differentiation of ES cells.

\section{Ectopic expression of Tcfl maintains self-renewal of ES cells in the absence of LIF}

To investigate the functions of Tcfs and Lef1 proteins, Tcfs and Lefl constructs cloned from RNA expressed in ES cells were stably introduced into ES cells (Fig. 2A). First, we tested which Tcfs and Lefl proteins regulated the stemness of ES cells using alkaline phosphatase (AP), a marker of self-renewal, staining (Fig. 2B and 2C). When LIF was removed from media, $\mathrm{AP}$ positive cells $\left(\mathrm{AP}^{+}\right)$ were remarkably reduced and the morphology was lost in ES cells expressing empty vector. Likewise, $\mathrm{AP}^{+}$decreased steadily in ES cells expressing Lef1, Tcf3, or Tcf4 (Fig. $2 \mathrm{C})$. In contrast, $\mathrm{AP}^{+}$was retained and the morphology remained intact in ES cells expressing Tcfl but not dominant-negative Tcf1 (TCF1-DN) even after the removal of LIF for 12 days (Fig. 2B). Moreover, ES cells expressing Tcfl were still stained with the antibody against SSEA1, a marker for proliferation (data not shown). These results suggested that Tcf1 may regulate the self-renewal of ES cells.

To further explore how Tcf1 regulates the self-renewal of ES cells, we checked the levels of core stem cell markers; Oct4, Sox 2 and Nanog upon Tcf1 overexpression at ES cells stage and Day 4 of differentiation. We found that during ES cells differentiation, the levels of Oct4, Sox2 and Nanog were reduced. However, upon overexpression of Tcfl, the reduction of stem cell markers was hindered. These effects were most predominantly seen in Nanog levels compared to Sox2 and Oct4 (Fig. 3A). We speculated that Tcfl is the major regulator for Nanog, therefore we focused on Nanog in subsequent experiments.

Nanog is vital for the self-renewal of ES cells $(7,8)$ and its promoter is regulated by $\operatorname{Tcf} 3(27,28)$. Since all Tcfs and Lef1 proteins bind to the same consensus sequence, we tested whether Tcfl regulates the expression of Nanog in days specific manner. We found that Nanog expression was reduced in control ES cells after day 4 of LIF depletion, whereas the expression of Tcfl inhibited the reduction in Nanog expression (Fig. 3B). These data indicate that ectopic Tcfl induced the self-renewal of ES cells by promoting Nanog expression.

To investigate whether Tcfl binds to Nanog promoter in practice, we performed a chromatin immunoprecipitation (ChIP) assay using an endogenous antibody for Tcfl, 
(A)
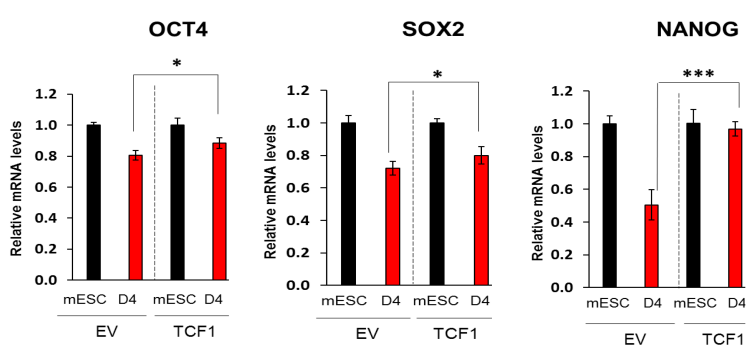

(D)

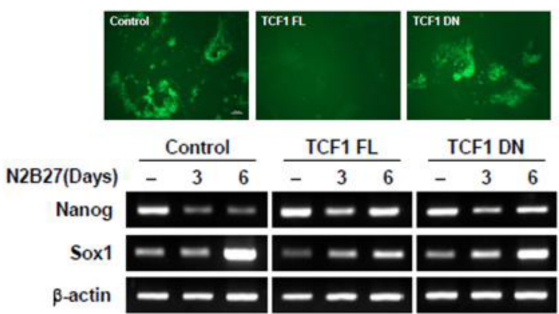

(B)

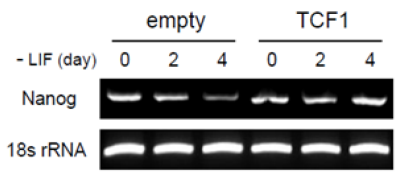

(C)

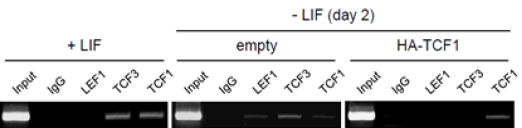

(E)

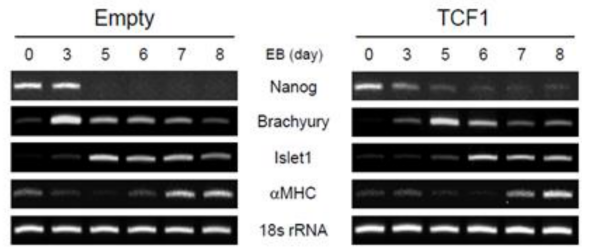

Fig. 3. Ectopic expression of Tcf1 maintains self-renewal and delays differentiation of ES cells. (A) mRNA expressions for Oct4, Sox 2 and Nanog at ES cells stage and Day 4 of differentiation upon TCF1 overexpression were determined by using qPCR. RNA was isolated from cells expressing empty vector (EV) or Tcf1-FL (TCF1) on specified stage. GAPDH was used for normalization. (B) The amount of Nanog transcript was measured by RT-PCR analysis. RNA was isolated from ES cells expressing control vector or Tcf1-FL on the days specified. 18s rRNA was used as a loading control. (C) ChIP assay was performed to examine the binding of Tcf1, Lef1, and Tcf3 on Nanog promoter. Samples for ChIP were isolated from ES cells grown with LIF and from ES cells expressing control vector or Tcf1-FL cultured for 2 days without LIF. For immunoprecipitiation, endogenous antibody against Tcf1, Lef1, or Tcf3 was used. (D) To differentiate into neural precursors, 46C ES cells expressing control vector, Tcf1-FL, or Tcf1-DN were plated for 6 days in N2B27 medium. GFP fluorescence showed that 46C ES cells expressing control vector or Tcf1-DN, but not Tcf1-FL effectively differentiated into neural precursor (upper panel). The amount of Nanog or Sox 1 transcript on the days indicated was measured via RT-PCR analysis (lower panel). $\beta$-actin was used as a loading control. (E) RNA samples were isolated from A6P10 ES cells expressing control vector or Tcf1-FL on the days indicated after EB formation. Self-renewal (Nanog), mesoderm (Brachyury), and cardiomyocyte (Islet1, $\alpha \mathrm{MHC}$ ) markers were used for RT-PCR analysis.

Tcf3, and Lef1 (Fig. 3C). In the presence of LIF, Tcf1 bound to the promoter of Nanog. Consistent with previous reports $(27,28)$, Tcf3 also interacted with Nanog promoter, suggesting that Tcf3 acts as an limiter of ES cell self-renewal. Since Lef1 protein is not expressed in the presence of LIF, no interaction between Lef1 and Nanog promoter was observed. Based on these data, we hypothesized that the occupancy of Tcfl on Nanog gene may be reduced during differentiation, which was demonstrated using a ChIP assay for Nanog gene after LIF removal for 2 days. As expected, the occupancy of Tcfl was reduced relative to that of Tcf3 and Lef1 (Fig. 3B). The protein levels of Tcf1, Tcf3, and Lef1 were the highest 2 days after LIF removal or after incubation with N2B27 media (Fig. 1B). Thus, the reduced binding of Tcf1 with Nanog promoter might be attributed to competition with Tcf3 or Lef1 protein. To support this possibility, ES cells stably expressing Tcfl were used in the ChIP assay. Interestingly, most of the Nanog promoter was occupied by Tcfl (Fig. 3B). Taken together, these results indicate that Tcf1 pro- motes the transcription of Nanog by binding with its promoter. The binding of Tcfl protein with Nanog promoter is prevented by the increasing levels of Tcf3 or Lef1 protein during differentiation.

\section{ES cell differentiation is prevented by Tcfl expression}

Nanog has been shown to be necessary for self-renewal and to prevent differentiation of ES cells in the absence of LIF $(7,8)$. Since ectopic expression of Tcf1 induced Nanog expression even after LIF depletion, we investigated whether overexpression of Tcfl inhibited normal differentiation. For differentiation into neural precursors, we used Soxl-GFP knock-in (46C) ES cells in which the differentiated cells are easily detected based on the GFP signal when cultured with N2B27 medium (33). In control 46C ES cells, GFP-positive cells appeared after 6 days of incubation in N2B27 medium. However, the ectopic expression of Tcfl, but not TCF1-DN, inhibited the emergence of GFP-positive cells (Fig. 3D, upper panel). Consistent with the GFP signal, the expression of Soxl, a 
marker of neural precursors, was not induced in 46C ES cells expressing Tcfl (Fig. 3D, lower panel). In addition, Nanog expression was not declined compared to the control cells after 6 days of incubation in N2B27 medium. These data indicate that overexpression of Tcfl results in the inhibition of differentiation into neural precursors via maintaining the levels of Nanog.

To determine whether the inhibition of differentiation by Tcfl overexpression occurred only in the case of differentiation into neural precursors, we further analyzed the cardiomyocyte differentiation via EB formation (Fig. 3D). While the expression of Nanog was diminished starting from day 5 in control $\mathrm{EB}$, it persisted even after 8 days in EB expressing Tcfl. Moreover, the expression of marker genes related to cardiomyocyte differentiation was delayed in EB-expressing Tcfl. These data show that the ectopic expression of Tcfl leads to delayed differentiation into cardiomyocytes. Overall, the overexpression of Tcfl prevented normal differentiation via maintaining the level of Nanog although it is possible that Tcfl may still regulate the genes related to differentiation.

\section{Knockdown of Lef1 inhibits differentiation of ES cells}

$\mathrm{AP}$ staining revealed that the ectopic expression of Lef1 did not induce self-renewal of ES cells. Interestingly, we also found that the expression of dominant-negative Lef1
(Lef1-DN) maintained ES cell self-renewal even after 12 days of LIF removal (Fig. 2C). In addition, endogenous Lefl protein was transiently expressed following differentiation (Fig. 1B). These results suggested that Lef1 may be related to differentiation. To test this possibility, the level of Lef1 was reduced by shRNA against $\beta$-cateninbinding domain of Lefl to knock down full-length Lefl but not dominant-negative Lef1. Two days after LIF removal, the expression of Lef1 protein was remarkably reduced by shRNA for Lef1 (Fig. 4A). In order to identify the effect of Lef1 reduction, 46C ES cells expressing shControl or shLefl were differentiated into neural precursors in N2B27 medium. Notably, 46C ES cells expressing shLef1 did not express Soxl-GFP compared with control after differentiation (Fig. 4B). Nevertheless, the level of Nanog in 46C ES cells expressing shLefl was reduced in the control after 7 days of differentiation (Fig. 4B, lower panel). These results indicate that knockdown of Lef1 inhibits differentiation of ES cells into neural precursors without upregulation of Nanog expression. Similar to shLef1, the overexpression of dominant negative Lef1 blocked the differentiation into neural precursors. We further tested whether ectopic expression of Lef1 enhanced the differentiation of ES cells. Surprisingly, overexpression of full-length Lef1 did not enhance differentiation, which was blocked, instead (Fig. 4C). This result may be ex-
(A)

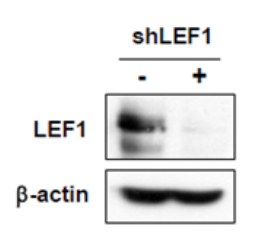

(C)

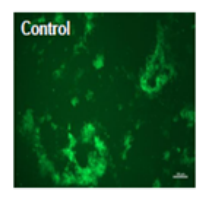

(B)

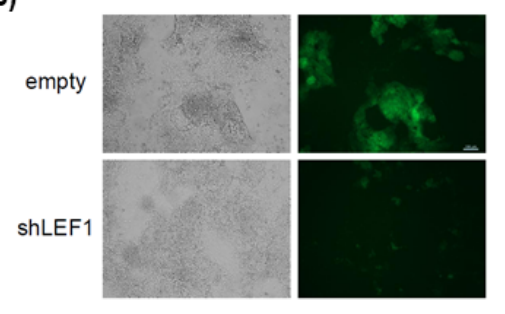

(D)
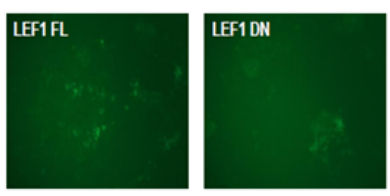
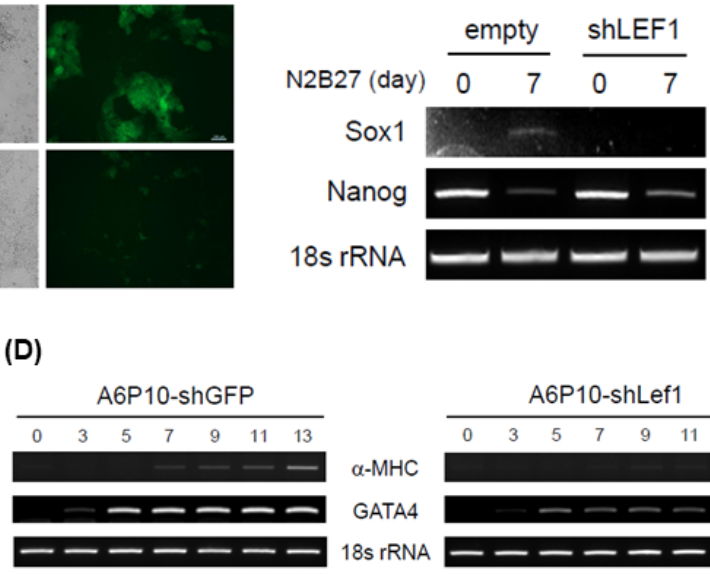

Fig. 4. Transient expression of Lef1 is required for proper differentiation. (A) A6P10 ES cells were infected with retroviruses expressing control or Lef1 shRNA, followed by selection using puromycin. After 2 days without LIF, the selected ES cells were harvested and analyzed by western blotting with anti-Lef1 antibody. $\beta$-actin was used as a loading control. (B) 46C ES cells expressing control shRNA or Lef1 shRNA were plated for 7 days in N2B27 medium. GFP fluorescence indicating a neural precursor was not induced in shLef1-46C cells (left panel). The amount of Nanog or Sox1 transcript was measured by RT-PCR analysis (right panel). (C) 46C ES cells were transfected with control vector, Lef1-FL, or Lef1-DN and then selected using Zeocin. 46C ES cells expressing each vector were plated for 7 days in N2B27 medium and GFP fluorescence was captured. (D) RNA samples were isolated from A6P10 ES cells expressing shGFP or shLef1 on the days indicated post-EB formation. Cardiomyocyte ( $\alpha-\mathrm{MHC}$ ) and endoderm (GATA4) markers and loading control (18s rRNA) were used for RT-PCR analysis. 


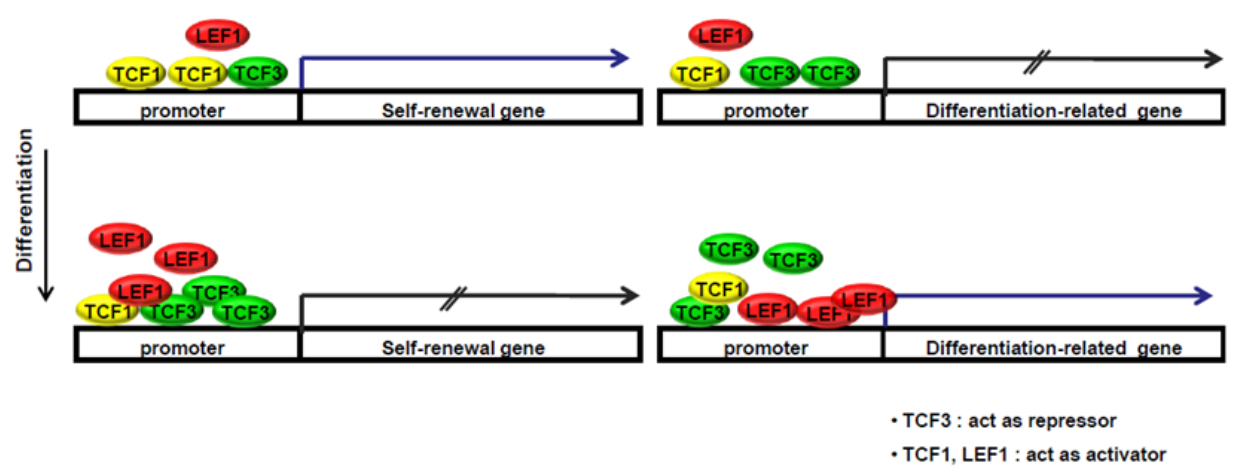

Fig. 5. A model describing the role of Tcfs and Lef1s in ES cells.

plained in part by the transient expression of endogenous Lef1 protein at the onset of differentiation (Fig. 1B). The effect of Lef1 reduction was also evaluated in the differentiation into cardiomyocytes. The expression of $\alpha$-MHC, a marker for cardiomyocytes, was attenuated in ES cells expressing shLefl. Since EB can also be partially differentiated into endoderm, we investigated the effect of shLef1 on endoderm differentiation. Knockdown of Lef1 reduced the expression of GATA4, an endoderm marker (Fig. 4D). These data show that the reduced Lefl expression attenuated the differentiation into cardiac mesoderm and endoderm. Taken together, these results suggest that the transient expression of Lef1 protein at the onset of differentiation is required for differentiation to three germ layers.

\section{Discussion}

Given that the effects of Wnt signaling vary, we speculated that the capacity of downstream effector molecules in the Wnt signaling pathway may differ depending on the status of ES cells. In this study, we found that the protein levels of Tcfs and Lef1 changed dynamically during the three types of differentiation and the expression pattern of each Tcfs and Lef1 varied (Fig. 1B). These results suggested that the individual Tcfs and Lef1 proteins may have a specific role in the regulation of self-renewal or differentiation of ES cells into specific lineages. Another interesting finding was that the expression patterns of Tcfs and Lef1 proteins varied, in part, from those of Tcfs and Lef1 transcripts. Especially, the level of Tcf3 protein declined sharply via unknown mechanism during EB formation while the level of transcript remained constant throughout differentiation (Fig. 1). The study of Tcfs and Lef1 1 function in ES cells has been limited to investigations at transcriptional level (27-30). Because Tcfs and Lef1 are regulated via unexpected degradation similar to post-transcriptional modification (26), the protein levels of
Tcfs/Lef1 must be investigated to define their expression patterns.

Tcf1, Lef1, and Tcf4 have been generally used without distinction due to their structural and functional similarity in vitro, while Tcf3 has been used separately. However, knockout studies showed that Tcfs and Lef1 have non-redundant functions in part (25). In addition, our data showed varied levels of Tcfs and Lef1 protein (Fig. 1B). Based on these data, we hypothesized that each Tcfs and Lef1 protein may have a differential role in self-renewal or differentiation of ES cells. We found that the overexpression of wild type Tcflcontributed to self-renewal (Fig. 2B), whereas knockdown of Tcfl had no effect on the undifferentiated state induced by LIF (data not shown). These results indicate that Tcfl is sufficient, but not necessary, to maintain self-renewal. As Tcfl protein level increased during differentiation (Fig. 1B), Tcf1 is possibly related to differentiation. To confirm this concern, further experiments are needed. Based on knockdown and ectopic expression of Lefl, we demonstrated that transient expression of Lefl at the onset of differentiation was required for normal differentiation (Fig. 4). Interestingly, canonical Wnt transcripts are expressed similar to Lef1 expression pattern $(19,20)$ and TOP reporter activity is increased at the early stage of differentiation (unpublished data). These data suggest that Lef1 may induce cell-autonomous stimulation of differentiation by canonical Wnts.

In summary, we identified distinct roles of Tcfl and Lefl in the regulation of self-renewal and differentiation of ES cells. Specific models illustrating the roles of Tcfs and Lef1 in the maintenance of stemness and regulation of differentiation are presented (Fig. 5). In undifferentiated ES cells, Tcf3 represses both self-renewal and differentiation-related genes, and Tcf1 mainly activates genes related to self-renewal to maintain stemness. During differentiation, increased Lef1 and Tcf3 may competitively bind to promoters of genes related to self-renewal and re- 
duce the binding of Tcfl on those promoters. In addition, increased levels of Lef1 may replace Tcf3 on gene promoters related to ES cell differentiation. In this study, we suggest that a balance between Tcfs and Lef1 is important for the modulation of self-renewal and differentiation in mouse embryonic stem cells.

\section{Acknowledgments}

This work was supported by the Basic Study and Interdisciplinary R\&D Foundation Fund of the University of Seoul (2019) for Eek-hoon Jho and Basic Science Research Program through the National Research Foundation of Korea (NRF) funded by the Ministry of Education (NRF-2017R1D1A1B03036097) for Hanjun Kim.

\section{Potential Conflict of Interest}

The authors have no conflicting financial interest.

\section{Supplementary Materials}

Supplementary data including one table can be found with this article online at http://pdf.medrang.co.kr/paper/ pdf/IJSC/IJSC-13-s20044.pdf.

\section{References}

1. Reubinoff BE, Pera MF, Fong CY, Trounson A, Bongso A. Embryonic stem cell lines from human blastocysts: somatic differentiation in vitro. Nat Biotechnol 2000;18:399-404

2. Thomson JA, Itskovitz-Eldor J, Shapiro SS, Waknitz MA, Swiergiel JJ, Marshall VS, Jones JM. Embryonic stem cell lines derived from human blastocysts. Science 1998;282: $1145-1147$

3. Smith AG. Embryo-derived stem cells: of mice and men. Annu Rev Cell Dev Biol 2001;17:435-462

4. Dahéron L, Opitz SL, Zaehres H, Lensch MW, Andrews PW, Itskovitz-Eldor J, Daley GQ. LIF/STAT3 signaling fails to maintain self-renewal of human embryonic stem cells. Stem Cells 2004;22:770-778

5. Nichols J, Zevnik B, Anastassiadis K, Niwa H, KleweNebenius D, Chambers I, Schöler H, Smith A. Formation of pluripotent stem cells in the mammalian embryo depends on the POU transcription factor Oct4. Cell 1998;95: 379-391

6. Avilion AA, Nicolis SK, Pevny LH, Perez L, Vivian N, Lovell-Badge R. Multipotent cell lineages in early mouse development depend on SOX2 function. Genes Dev 2003; 17:126-140

7. Chambers I, Colby D, Robertson M, Nichols J, Lee S, Tweedie S, Smith A. Functional expression cloning of Nanog, a pluripotency sustaining factor in embryonic stem cells. Cell 2003;113:643-655
8. Mitsui K, Tokuzawa Y, Itoh H, Segawa K, Murakami M, Takahashi K, Maruyama M, Maeda M, Yamanaka S. The homeoprotein Nanog is required for maintenance of pluripotency in mouse epiblast and ES cells. Cell 2003;113:631642

9. Niwa H, Miyazaki J, Smith AG. Quantitative expression of Oct-3/4 defines differentiation, dedifferentiation or self-renewal of ES cells. Nat Genet 2000;24:372-376

10. Reya $\mathrm{T}$, Clevers $\mathrm{H}$. Wnt signalling in stem cells and cancer. Nature 2005;434:843-850

11. Merrill BJ. Wnt pathway regulation of embryonic stem cell self-renewal. Cold Spring Harb Perspect Biol 2012;4:a007971

12. Aubert J, Dunstan H, Chambers I, Smith A. Functional gene screening in embryonic stem cells implicates Wnt antagonism in neural differentiation. Nat Biotechnol 2002;20: 1240-1245

13. Haegele L, Ingold B, Naumann H, Tabatabai G, Ledermann $\mathrm{B}$, Brandner S. Wnt signalling inhibits neural differentiation of embryonic stem cells by controlling bone morphogenetic protein expression. Mol Cell Neurosci 2003;24:696708

14. Kielman MF, Rindapää $M$, Gaspar C, van Poppel N, Breukel C, van Leeuwen S, Taketo MM, Roberts S, Smits R, Fodde R. Apc modulates embryonic stem-cell differentiation by controlling the dosage of beta-catenin signaling. Nat Genet 2002;32:594-605

15. Sato N, Meijer L, Skaltsounis L, Greengard P, Brivanlou AH. Maintenance of pluripotency in human and mouse embryonic stem cells through activation of Wnt signaling by a pharmacological GSK-3-specific inhibitor. Nat Med 2004; 10:55-63

16. Kirby LA, Schott JT, Noble BL, Mendez DC, Caseley PS, Peterson SC, Routledge TJ, Patel NV. Glycogen synthase kinase 3 (GSK3) inhibitor, SB-216763, promotes pluripotency in mouse embryonic stem cells. PLoS One 2012;7:e39329

17. Dravid G, Ye Z, Hammond H, Chen G, Pyle A, Donovan $\mathrm{P}, \mathrm{Yu} \mathrm{X}$, Cheng L. Defining the role of Wnt/beta-catenin signaling in the survival, proliferation, and self-renewal of human embryonic stem cells. Stem Cells 2005;23:1489-1501

18. Kwon C, Arnold J, Hsiao EC, Taketo MM, Conklin BR, Srivastava D. Canonical Wnt signaling is a positive regulator of mammalian cardiac progenitors. Proc Natl Acad Sci USA 2007;104:10894-10899

19. Lindsley RC, Gill JG, Kyba M, Murphy TL, Murphy KM. Canonical Wnt signaling is required for development of embryonic stem cell-derived mesoderm. Development 2006; 133:3787-3796

20. Naito AT, Shiojima I, Akazawa H, Hidaka K, Morisaki T, Kikuchi A, Komuro I. Developmental stage-specific biphasic roles of Wnt/beta-catenin signaling in cardiomyogenesis and hematopoiesis. Proc Natl Acad Sci USA 2006;103: 19812-19817

21. Otero JJ, Fu W, Kan L, Cuadra AE, Kessler JA. Beta-catenin signaling is required for neural differentiation of embryonic stem cells. Development 2004;131:3545-3557

22. Kim H, Kim S, Song Y, Kim W, Ying QL, Jho EH. Dual 
function of Wnt signaling during neuronal differentiation of mouse embryonic stem cells. Stem Cells Int 2015;2015: 459301

23. Cadigan KM, Waterman ML. TCF/LEFs and Wnt signaling in the nucleus. Cold Spring Harb Perspect Biol 2012;4: a007906

24. Arce L, Yokoyama NN, Waterman ML. Diversity of LEF/ TCF action in development and disease. Oncogene 2006;25: $7492-7504$

25. Hoppler S, Kavanagh CL. Wnt signalling: variety at the core. J Cell Sci 2007;120(Pt 3):385-393

26. Hurlstone A, Clevers H. T-cell factors: turn-ons and turnoffs. EMBO J 2002;21:2303-2311

27. Cole MF, Johnstone SE, Newman JJ, Kagey MH, Young RA. Tcf3 is an integral component of the core regulatory circuitry of embryonic stem cells. Genes Dev 2008;22:746755

28. Pereira L, Yi F, Merrill BJ. Repression of Nanog gene transcription by Tcf3 limits embryonic stem cell self-renewal. Mol Cell Biol 2006;26:7479-7491
29. Tam WL, Lim CY, Han J, Zhang J, Ang YS, Ng HH, Yang H, Lim B. T-cell factor 3 regulates embryonic stem cell pluripotency and self-renewal by the transcriptional control of multiple lineage pathways. Stem Cells 2008;26:2019-2031

30. Yi F, Pereira L, Merrill BJ. Tcf3 functions as a steady-state limiter of transcriptional programs of mouse embryonic stem cell self-renewal. Stem Cells 2008;26:1951-1960

31. Zhang Y, Zhu Z, Ding H, Wan S, Zhang X, Li Y, Ji J, Wang $\mathrm{X}$, Zhang $M$, Ye SD. $\beta$-catenin stimulates Tcf7ll degradation through recruitment of casein kinase 2 in mouse embryonic stem cells. Biochem Biophys Res Commun 2020; 524:280-287

32. Ye S, Zhang T, Tong C, Zhou X, He K, Ban Q, Liu D, Ying QL. Depletion of Tcf3 and Lef1 maintains mouse embryonic stem cell self-renewal. Biol Open 2017;6:511-517

33. Ying QL, Stavridis M, Griffiths D, Li M, Smith A. Convertsion of embryonic stem cells into neuroectodermal precursors in adherent monoculture. Nat Biotechnol 2003;21: 183-186 